\title{
Further Results on Distributed Secondary Control in Microgrids
}

\author{
Hedi Bouattour, John W. Simpson-Porco, Florian Dörfler, and Francesco Bullo
}

\begin{abstract}
This work proposes several analysis and design results for primary droop control and secondary control in inductive microgrids. Building on our recent work, we study the problem of set point design in primary-controlled microgrids, and provide a choice of droop coefficients leading to the desired power flows. We then compare and contrast two distributed secondary-control schemes, and extend them to the case where only a fraction of inverters cooperates in regulating the network frequency. We show that both these secondary-control schemes achieve frequency regulation in the presence of network losses.
\end{abstract}

\section{INTRODUCTION}

Microgrids are low-voltage electrical distribution networks, heterogeneously composed of distributed generation, storage, load, and managed autonomously from the larger primary network. Microgrids are able to connect to the wide area electric power system through a Point of Common Coupling (PCC), but are also able to "island" themselves and operate independently [1], [2]. Energy generation within a microgrid can be highly heterogeneous, including photovoltaics, wind, geothermal, micro-turbines, etc. Many of these sources generate either variable frequency AC power or DC power, and are interfaced with a synchronous AC grid via power electronic DC/AC inverters. In islanded operation, it is through these inverters that actions must be taken to ensure synchronization, security, power balance and load sharing in the network [3].

The so-called primary droop controllers have been used successfully to achieve these tasks, see [3]-[9]. For inductive lines, the controller balances the active power demands in the network by instantaneously changing the frequency $\omega_{i}$ of the voltage signal at the $i^{\text {th }}$ inverter according to

$$
\omega_{i}=\omega^{*}-n_{i}\left(P_{\mathrm{e}, i}-P_{i}^{*}\right),
$$

where $\omega^{*}$ is a rated frequency, $P_{\mathrm{e}, i}$ is the active electrical power injection at node $i$, and $P_{i}^{*}$ is the nominal active power injection. The parameter $n_{i}>0$ is referred to as the droop coefficient. Despite forming the foundation for the operation of parallel inverters, primary-droop-controlled networks of inverters and loads have only recently been subject to a rigorous nonlinear analysis. In the recent work [10], the authors presented a necessary and sufficient condition for the existence of a unique and locally exponentially stable steady

This work was supported in part by the National Science Foundation NSF CNS-1135819 and by the National Science and Engineering Research Council of Canada.

Hedi Bouattour, J. W. Simpson-Porco, F. Dörfler and F. Bullo are with the Center for Control, Dynamical Systems and Computation, University of California at Santa Barbara. Email: hebouattour@gmail.com, \{johnwsimpsonporco, dorfler, bullo\}@engineering.ucsb.edu. state of a droop-controlled network. Moreover, a secondarycontrol scheme was devised - termed the distributedaveraging $P I$ controller — which dynamically regulates the network frequency to a nominal value while maintaining a proportional sharing of power among the inverters. These results are foundational to the present work, and are reviewed in Section II.

Conservative conditions for stability are presented in [11] for all-to-all networks controlled by droop-like controllers. In [12] state feedback is combined with a decentralized LMI strategy to ensure stabilization and frequency regulation, while [13] studies the performance of centralized and decentralized frequency-control algorithms based on integral action. In [14] a secondary-control scheme based on all-toall inverter frequency averaging is proposed. These works all represent variations on the theme of secondary control, but share the common disadvantage that the steady-state power injections after the frequency is restored can be quite disparate when compared to the power injections before, unless the network topology, weights, and controller gains are carefully manipulated. That is, the additional integral action disrupts any fair sharing of power between the distributed generators established by the primary stabilizing controller.

The contributions of this work are as follows. Starting from our previous analysis in [10], we present several novel analysis and design results for frequency-droop and secondary control in inductive microgrids. First, in Section III we characterize the set of droop coefficients which leads to a desired configuration of power flows for primary-controlled networks. In Section IV-A we present necessary and sufficient stability conditions for the secondary-control scheme proposed in [14], while in Section IV-B we partially extend our secondary-control results to lossy networks. Section IV-C extends our secondary-control results to the case where only a fraction of inverters participate in frequency regulation. Finally, Section V contains simulations illustrating some of our results.

The remainder of the paper is organized as follows. In Sections II-II-C we review our previous works on primary and distributed secondary control. The remaining sections of the paper contain our main results, with some comments and outlook at the conclusion of the document.

\section{Problem Setup, Droop Control And DAPI CONTROL}

\section{A. Preliminaries and Notation}

Sets, vectors and functions: Given a finite set $\mathcal{V}$, let $|\mathcal{V}|$ denote its cardinality. Given an index set $\mathcal{I}$ and a real valued 1D-array $\left\{x_{1}, \ldots, x_{|\mathcal{I}|}\right\}, \operatorname{diag}\left(\left\{x_{i}\right\}_{i \in \mathcal{I}}\right) \in \mathbb{R}^{|\mathcal{I}| \times|\mathcal{I}|}$ is the associated diagonal matrix. We denote the $n \times n$ identity 
matrix by $I_{n}$. Let $\mathbf{1}_{n}$ and $\mathbf{0}_{n}$ be the $n$-dimensional vectors of all ones and all zeros, and let $\mathbf{1}_{n}^{\perp} \triangleq\left\{x \in \mathbb{R}^{n} \mid \mathbf{1}_{n}^{T} x=0\right\}$ be the subspace of $\mathbb{R}^{n}$ orthogonal to $\mathbf{1}_{n}$. We will drop the subscripts from $I_{n}, \mathbf{1}_{n}$ and $\mathbf{0}_{n}$ when the dimensions are clear from context. For a finite partitioned set $\mathcal{V}=\mathcal{V}_{L} \cup \mathcal{V}_{I}$, a vector $x \in \mathbb{R}^{|\mathcal{V}|}$ inherits the partitioning as $x=\left(x_{L}, x_{I}\right)$, where $x_{L} \in \mathbb{R}^{n_{L}}$ and $x_{I} \in \mathbb{R}^{n_{I}}$. For $x \in \mathbb{R}^{n}$, let $\sin (x) \triangleq$ $\left(\sin \left(x_{1}\right), \ldots, \sin \left(x_{n}\right)\right)^{T} \in \mathbb{R}^{n}$

Algebraic graph theory: We denote by $G(\mathcal{V}, \mathcal{E}, A)$ an undirected and weighted graph, where $\mathcal{V}$ is the set of nodes, $\mathcal{E} \subseteq \mathcal{V} \times \mathcal{V}$ is the set of edges, and $A \in \mathbb{R}^{|\mathcal{V}| \times|\mathcal{V}|}$ is the adjacency matrix. If a number $\ell \in\{1, \ldots,|\mathcal{E}|\}$ and an arbitrary direction is assigned to each edge $\{i, j\} \in \mathcal{E}$, the node-edge incidence matrix $B \in \mathbb{R}^{|\mathcal{V}| \times|\mathcal{E}|}$ is defined component-wise as $B_{k \ell}=1$ if node $k$ is the sink node of edge $\ell$ and as $B_{k \ell}=-1$ if node $k$ is the source node of edge $\ell$, with all other elements being zero. For $x \in \mathbb{R}^{|\mathcal{V}|}, B^{T} x \in \mathbb{R}^{|\mathcal{E}|}$ is the vector with components $x_{i}-x_{j}$, with $\{i, j\} \in \mathcal{E}$. If $\mathcal{A} \triangleq \operatorname{diag}\left(\left\{a_{i j}\right\}_{\{i, j\} \in \mathcal{E}}\right) \in \mathbb{R}^{|\mathcal{E}| \times|\mathcal{E}|}$ is the diagonal matrix of edge weights, then the positive semidefinite Laplacian matrix is given by $L=B \mathcal{A} B^{T}$. If the graph is connected, then $\operatorname{ker}\left(B^{T}\right)=\operatorname{ker}(L)=\operatorname{span}\left(\mathbf{1}_{|\mathcal{V}|}\right)$, and $\operatorname{ker}(B)=\emptyset$ for acyclic graphs. In this case, for every $x \in \mathbf{1}_{|\mathcal{V}|}^{\perp}$, that is, $\sum_{i \in \mathcal{V}} x_{i}=0$, there exists a unique $\xi \in \mathbb{R}^{|\mathcal{E}|}$ satisfying Kirchoff's Current Law (KCL) $x=B \xi$ [15], [16]. The vector $x$ is interpreted as nodal injections, with $\xi$ being the associated flows along edges. We denote by $B^{\dagger} \triangleq\left(B^{T} B\right)^{-1} B^{T}$ the pseudoinverse of $B$.

Geometry on the $n$-torus: The set $\mathbb{S}^{1}$ denotes the unit circle, an angle is a point $\theta \in \mathbb{S}^{1}$, and an arc is a connected subset of $\mathbb{S}^{1}$. With a slight abuse of notation, let $\left|\theta_{1}-\theta_{2}\right|$ denote the geodesic distance between two angles $\theta_{1}, \theta_{2} \in \mathbb{S}^{1}$. The $n$-torus $\mathbb{T}^{n}=\mathbb{S}^{1} \times \cdots \times \mathbb{S}^{1}$ is the Cartesian product of $n$ unit circles. For $\gamma \in[0, \pi / 2[$ and a given graph $G(\mathcal{V}, \mathcal{E}, \cdot)$, let $\Delta_{G}(\gamma)=\left\{\theta \in \mathbb{T}^{|\mathcal{V}|}: \max _{\{i, j\} \in \mathcal{E}}\left|\theta_{i}-\theta_{j}\right| \leq \gamma\right\}$ be the closed set of angle arrays $\theta=\left(\theta_{1}, \ldots, \theta_{n}\right)$ with neighboring angles $\theta_{i}$ and $\theta_{j},\{i, j\} \in \mathcal{E}$ no further than $\gamma$ apart.

\section{B. droop-controlled inverters in islanded microgrids}

For our purposes, a microgrid is a linear AC circuit with purely inductive admittance matrix $Y \in j \mathbb{R}^{n \times n}$. The associated connected, undirected, and complex-weighted graph is $G(\mathcal{V}, \mathcal{E}, A)$ with node set (or buses) $\mathcal{V}=\{1, \ldots, n\}$, edge set (or branches) $\mathcal{E} \subset \mathcal{V} \times \mathcal{V}$, and symmetric edge weights (or admittances) $a_{i j}=-Y_{i j}=-Y_{j i} \in \mathbb{C}$ for every branch $\{i, j\} \in \mathcal{E}$, where $Y$ is the bus admittance matrix. We partition the set of buses as $\mathcal{V}=\mathcal{V}_{L} \cup \mathcal{V}_{I}$, corresponding to the loads and inverters. For simplicity, we denote the respective sizes of $\mathcal{V}, \mathcal{V}_{L}$ and $\mathcal{V}_{I}$ by $n, n_{L}$ and $n_{I}$. To each bus $i \in \mathcal{V}$, we associate an active electrical power injection $P_{\mathrm{e}, i} \in \mathbb{R}$ and the phasor voltage variable $V_{i}=E_{i} e^{\sqrt{-1} \theta_{i}}$ corresponding to the magnitude $E_{i}>0$ and the phase shift $\theta_{i} \in \mathbb{S}$ of a harmonic voltage solution to the AC power flow equations. The active electrical power $P_{\mathrm{e}, i} \in \mathbb{R}$ injected into the network at node $i \in\{1, \ldots, n\}$ is then given by [17]

$$
P_{\mathrm{e}, i}(\theta)=\sum_{j=1}^{n} E_{i} E_{j}\left|Y_{i j}\right| \sin \left(\theta_{i}-\theta_{j}\right)
$$

Each load $i \in \mathcal{V}_{L}$ demands a constant amount of active power $P_{i}^{*}$ and must satisfy the power balance equation

$$
0=P_{i}^{*}-P_{\mathrm{e}, i}(\theta), \quad i \in \mathcal{V}_{L} .
$$

The frequency of each inverter $i \in \mathcal{V}_{I}$ is controlled according to the frequency droop control law (1), which as shown in [10] can be formulated as

$$
D_{i} \dot{\theta}_{i}=P_{i}^{*}-P_{\mathrm{e}, i}(\theta)+u_{i}(t), \quad i \in \mathcal{V}_{I},
$$

where $D_{i} \triangleq n_{i}^{-1}$ is the (inverse) droop coefficient, $u_{i}$ : $\mathbb{R}_{\geq 0} \rightarrow \mathbb{R}$ is a secondary-control input, and $P_{i}^{*} \in\left[0, \bar{P}_{i}\right]$ is a nominal injection, where $\bar{P}_{i}$ is the rating (power limit) of inverter $i$. In this equation, $\dot{\theta}_{i}=\omega_{i}-\omega^{*}$ is the deviation of the frequency from the nominal value $\omega^{*}$, cf. (1).

By summing over all equations (3)-(4) without secondarycontrol input $u_{i}=0$ for all $i \in \mathcal{V}_{I}$, it can be verified that if the closed-loop system possesses a frequency-synchronized solution $\dot{\theta}_{i}(t)=\omega_{\text {sync }} \in \mathbb{R}$ for all $i \in\{1, \ldots, n\}$, then the explicit synchronization frequency $\omega_{\text {sync }}$ is given by the scaled power imbalance

$$
\omega_{\text {sync }}=\omega_{\text {avg }} \triangleq \frac{\sum_{j \in \mathcal{V}} P_{i}^{*}}{\sum_{j \in \mathcal{V}_{I}} D_{j}} .
$$

By transforming to a rotating coordinate frame $\theta(t) \mapsto \theta(t)-$ $\omega_{\text {avg }} t(\bmod 2 \pi)$, a frequency-synchronized solution of $(3)$ (4) is equivalent to an equilibrium of

$$
\begin{array}{rlrl}
0 & =\widetilde{P}_{i}-P_{e, i}(\theta), & i & \in \mathcal{V}_{L}, \\
D_{i} \dot{\theta}_{i} & =\widetilde{P}_{i}-P_{e, i}(\theta), & i \in \mathcal{V}_{I},
\end{array}
$$

where $\widetilde{P}_{i}=P_{i}^{*}$ for $i \in \mathcal{V}_{L}$, and $\widetilde{P}_{i}=P_{i}^{*}-D_{i} \omega_{\text {avg }}$ for $i \in \mathcal{V}_{I}$. Using the incidence matrix $B$ of the graph describing the circuit, equilibria of (6a)-(6b) satisfy [10]

$$
\widetilde{P}=B \mathcal{A} \sin \left(B^{T} \theta^{*}\right)
$$

where $\mathcal{A}=\operatorname{diag}\left(\left\{E_{i} E_{j}\left|Y_{i j}\right|\right\}_{\{i, j\} \in \mathcal{E}}\right)$ and $\widetilde{P}=\left(\widetilde{P}_{1}, \ldots, \widetilde{P}_{n}\right)$. For acyclic networks, the following result gives a necessary and sufficient condition for the existence of an exponentially stable equilibrium of (6a)-(6b), and hence a synchronized solution of the closed-loop droop control system (3)-(4) [10, Theorem 2].

Theorem 2.1: (Existence and Stability of Sync'd Solution). Consider the frequency-droop-controlled system (3)(4) without secondary-control input $u_{i}=0$ for all $i \in \mathcal{V}_{I}$ and defined on an acyclic network with node-edge incidence matrix $B$. Let $\xi \in \mathbb{R}^{|\mathcal{E}|}$ be the unique vector of edge power flows satisfying KCL, given by $\xi=B^{\dagger} \widetilde{P}$. The following two statements are equivalent:

(i) Synchronization: There exists an arc length $\gamma \in$ $[0, \pi / 2[$ such that the closed-loop system (3)-(4) possesses a locally exponentially stable and unique ${ }^{\dagger}$ synchronized solution $t \mapsto \theta^{*}(t) \in \Delta_{G}(\gamma)$ for all $t \geq 0$;

(ii) Flow Feasibility: The power flow is feasible, i.e.,

$$
\Gamma \triangleq\left\|\mathcal{A}^{-1} \xi\right\|_{\infty}<1
$$

${ }^{\dagger}$ Modulo the rotational symmetry inherent in the model. 
If the equivalent statements (i) and (ii) hold true, then the quantities $\Gamma \in[0,1[$ and $\gamma \in[0, \pi / 2[$ are related uniquely via $\Gamma=\sin (\gamma)$, the synchronized solution satisfies $\theta^{*}(t)=\theta_{0}+\left(\omega_{\text {sync }} t \mathbf{1}_{n}\right)(\bmod 2 \pi)$ for some $\theta_{0} \in \Delta_{G}(\gamma)$, where $\omega_{\text {sync }}=\omega_{\text {avg }}$, and the synchronized angular differences satisfy $\sin \left(B^{T} \theta^{*}\right)=\mathcal{A} \xi$.

\section{Distributed-Averaging PI-control by Simpson-Porco et al.}

From Theorem 2.1, the droop controller (4) assures that the closed-loop system (3)-(4) without secondary-control input synchronizes to a constant frequency $\omega_{\text {sync }}$, given by the scaled power imbalance (5). The purpose of the secondarycontrol input $u_{i}$ in (4) is to enforce $\omega_{\text {avg }}=0$. That is, the network frequency is maintained at the nominal value. Equivalently, the secondary-control input needs to transform the primary-controlled system (3)-(4) into the equivalent system (6a)-(6b) in a rotating frame.

Together with the primary proportional droop controller (4), the following distributed-averaging proportional-integral (DAPI) controller has been proposed by the authors in [10]:

$$
u_{i}=-p_{i}, \quad k_{i} \dot{p}_{i}=D_{i} \dot{\theta}_{i}-\sum_{j \in \mathcal{V}_{I}} L_{\mathrm{c}, i j}\left(\frac{p_{i}}{D_{i}}-\frac{p_{j}}{D_{j}}\right) \text {. }
$$

Here, $L_{\mathrm{c}}$ is the Laplacian matrix of a connected communication graph between inverters, $p_{i} \in \mathbb{R}$ is an auxiliary power variable and $k_{i}>0$ is a gain, for each $i \in \mathcal{V}_{I}$. The resulting closed-loop system is then given by

$$
\begin{aligned}
0 & =P_{i}^{*}-P_{\mathrm{e}, i}(\theta), & & i \in \mathcal{V}_{L}, \\
D_{i} \dot{\theta}_{i} & =P_{i}^{*}-p_{i}-P_{\mathrm{e}, i}(\theta), & i & \in \mathcal{V}_{I}, \\
k_{i} \dot{p}_{i} & =D_{i} \dot{\theta}_{i}-\sum_{j \in \mathcal{V}_{I}} L_{\mathrm{c}, i j}\left(\frac{p_{i}}{D_{i}}-\frac{p_{j}}{D_{j}}\right), & & i \in \mathcal{V}_{I} .
\end{aligned}
$$

The following result summarizes the stability of the closedloop system (10) for an acyclic network [10, Theorem 8].

Theorem 2.2: (Stability of DAPI-Controlled Network). Consider an acyclic network of droop-controlled inverters and loads in which the inverters can communicate through the weighted graph $G_{c}$, as described by the closed-loop system (10) with parameters $k_{i}>0, P_{i}^{*} \in\left[0, \bar{P}_{i}\right]$, and $D_{i}>0$ for $i \in \mathcal{V}_{I}$, and connected communication Laplacian $L_{\mathrm{c}} \in \mathbb{R}^{n_{I} \times n_{I}}$. The following two statements are equivalent:

(i) Stability of Droop Controller: The droop control stability condition (8) holds;

(ii) Stability of DAPI Controller: There exists an arc length $\gamma \in[0, \pi / 2[$ such that the system (10) possesses a locally exponentially stable and unique ${ }^{\dagger}$ equilibrium $\left(\theta^{*}, p^{*}\right) \in \Delta_{G}(\gamma) \times \mathbb{R}^{n_{I}}$.

If the equivalent statements (i) and (ii) hold true, then the unique equilibrium is given as in Theorem 2.1 (ii), along with $p_{i}^{*}=D_{i} \omega_{\text {avg }}$ for $i \in \mathcal{V}_{I}$. Moreover, if the droop coefficients are selected proportionally, then the DAPI controller (9) preserves the proportional power sharing property of the primary droop controller.

In [10], we also show that the DAPI controller (9) is robust to unmodeled voltage dynamics.

\footnotetext{
${ }^{\dagger}$ Modulo the rotational symmetry inherent in the model.
}

\section{Power Flow Design Using Droop CoefFicients}

The droop coefficients are conventionally selected in proportion to the power ratings $\bar{P}_{i}$ of the inverters, with this choice leading to a proportional sharing power among the inverters [10]. This choice of parameters eliminates all remaining degrees of freedom in the controller, as all droop coefficients are then determined up to an arbitrary positive constant.

In this section we focus on primary control and address the following "controllability" type question: how much can one influence the steady-state power injections of the inverters (or the steady-state branch flows of the network) through the choice of droop coefficients? In particular, given a vector of desired steady-state power injections $P_{I}^{\text {set }} \in \mathbb{R}^{n_{I}}$ for the inverters, when and how can one select the droop coefficients to generate these injections in steady state? We require the following standing assumption.

\section{Assumption 1: (Actuation Assumptions).}

(i) Nominal Injection: $P_{i}^{*}=\bar{P}_{i}$ for each $i \in \mathcal{V}_{I}$. (ii) Serviceable Load: $-\sum_{j \in \mathcal{V}_{I}} \bar{P}_{j}<\sum_{j \in \mathcal{V}_{L}} P_{j}^{*} \leq 0$;

From (5), Assumption 1 (i)-(ii) together guarantee that $\omega_{\text {avg }} \neq 0$ (specifically, that $\omega_{\text {avg }}>0$ ), and hence that the droop coefficients influence the steady-state inverter power injections $P_{\mathrm{e}, i}=P_{i}^{*}-\omega_{\mathrm{avg}} D_{i}, i \in \mathcal{V}_{I}$. One could formulate alternate assumptions such that $\omega_{\text {avg }}<0$, and all results which follow would go through with minor modifications.

Definition 1: (Power Injection Setpoint). A vector $P^{\text {set }} \in \mathbb{R}^{n}$ is a power injection set-point if $P^{\text {set }} \in \mathbf{1}_{n}^{\perp}$ and $P_{i}^{\text {set }}=P_{i}^{*}$ for $i \in \mathcal{V}_{L}$.

This is simply that a set-point is a point of power balance, and that we have no say in the power demand at the loads.

Definition 2: $\left(\boldsymbol{\gamma}\right.$-Feasible Setpoint). Let $P^{\text {set }}=$ $\left(P_{L}^{*}, P_{I}^{\text {set }}\right)^{T}$ be a power injection set-point, with $\xi^{\text {set }} \in \mathbb{R}^{|\mathcal{E}|}$ being the associated branch flows $\xi^{\text {set }}=B^{\dagger} P^{\text {set }}$, and let $\gamma \in\left[0, \pi / 2\left[\right.\right.$. The power injection set-point $P^{\text {set }}$

(i) satisfies the nodal actuation constraint if $P_{i}^{\text {set }}<\bar{P}_{i}$, $i \in \mathcal{V}_{I}$

(ii) is $\gamma$-feasible if it satisfies the nodal actuation constraint and $\left\|\mathcal{A}^{-1} \xi^{\text {set }}\right\|_{\infty} \leq \sin (\gamma)$.

The following result characterizes possible selections of droop coefficients that lead to a desired set-point.

Theorem 3.1: (Power Injection Set-Point Design). Let $P^{*} \in \mathbb{R}^{n}$ satisfy Assumption 1 , let $P^{\text {set }}$ be a power injection set-point, and let $\gamma \in[0, \pi / 2[$. The following statements are equivalent:

(i) Coefficient Selection: There exists a selection of droop coefficients such that the steady-state power injections satisfy $P_{\mathrm{e}}\left(\theta^{*}\right)=P^{\text {set }}$, with $\theta^{*} \in \Delta_{G}(\gamma)$;

(ii) Set-Point Feasibility: $P^{\text {set }}$ is a $\gamma$-feasible power injection set-point.

For any $\beta>0$, the choice of droop coefficients

$$
D_{i}=\beta\left(\bar{P}_{i}-P_{i}^{\text {set }}\right)>0, \quad i \in \mathcal{V}_{I},
$$

leads to the desired property of the synchronized steady state.

Proof: (i) $\Rightarrow$ (ii): Let $\left\{D_{i}\right\}_{i \in \mathcal{V}_{I}}$ be an appropriate selection of droop coefficients, satisfying $D_{i}>0$ for each 
$i \in \mathcal{V}_{I}$. Since $P_{\mathrm{e}} \in \mathbf{1}_{n}^{\perp}, P^{\text {set }} \in \mathbf{1}_{n}^{\perp}$ and hence $P^{\text {set }}$ is a power injection set-point. We first show that $P^{\text {set }}$ satisfies the nodal actuation constraint. In steady state, it holds for each $i \in \mathcal{V}_{I}$ that

$$
P_{\mathrm{e}, i}(\theta)=P_{i}^{\mathrm{set}}=\bar{P}_{i}-\omega_{\mathrm{avg}} D_{i}
$$

Under Assumption 1, $\omega_{\text {avg }}>0$, and hence $P_{i}^{\text {set }}-P_{i}^{*}<0$ for each $i \in \mathcal{V}_{I}$. Hence, $P^{\text {set }}$ satisfies the nodal actuation constraint. Since $\theta^{*} \in \Delta_{G}(\gamma)$, we have that $\left\|\mathcal{A}^{-1} \xi^{\text {set }}\right\|_{\infty} \leq$ $\sin (\gamma)$, and hence $P^{\text {set }}$ is $\gamma$-feasible.

(ii) $\Rightarrow$ (i): Suppose now that $P^{\text {set }}$ is a $\gamma$-feasible power injection set-point. Then for each $i \in \mathcal{V}_{I}, \bar{P}_{i}>P_{i}^{\text {set }}$. For $\beta>0$, consider then the selection of droop coefficients $D_{i}=\beta\left(P_{i}^{*}-P_{i}^{\text {set }}\right)>0$. For each $i \in \mathcal{V}_{I}$ we calculate that

$$
\begin{aligned}
P_{\mathrm{e}, i} & =P_{i}^{*}-\omega_{\mathrm{avg}} D_{i}=P_{i}^{*}-\frac{\mathbf{1}_{n}^{T} P^{*} \cdot \beta\left(P_{i}^{*}-P_{i}^{\mathrm{set}}\right)}{\beta \mathbf{1}_{n_{I}}^{T}\left(P_{I}^{*}-P_{I}^{\mathrm{set}}\right)} \\
& =P_{i}^{*}-\left(\frac{\mathbf{1}_{n}^{T} P^{*}}{\mathbf{1}_{n_{I}}^{T} P_{I}^{*}-\mathbf{1}_{n_{I}}^{T} P_{I}^{\mathrm{set}}}\right)\left(P_{i}^{*}-P_{i}^{\mathrm{set}}\right) \\
& =P_{i}^{*}-\left(\frac{\mathbf{1}_{n}^{T} P^{*}}{\mathbf{1}_{n}^{T} P^{*}}\right)\left(P_{i}^{*}-P_{i}^{\mathrm{set}}\right)=P_{i}^{\mathrm{set}},
\end{aligned}
$$

where we have used the fact that $\mathbf{1}_{n_{I}}^{T} P_{I}^{\text {set }}=-\mathbf{1}_{n_{L}}^{T} P_{L}^{*}$. Since $P_{\mathrm{e}, i}=P_{i}^{*}=P_{i}^{\text {set }}$ for each $i \in \mathcal{V}_{L}$, we have that $P_{\mathrm{e}}=$ $P^{\text {set }}$. Since $P^{\text {set }}$ is $\gamma$-feasible, the steady state for the droopcontrolled network is well defined and locally exponentially stable, and in particular $\theta^{*} \in \Delta_{G}(\gamma)$.

Note that for parallel inverters, designing $P_{I}^{\text {set }}$ is equivalent to designing the branch flows since $\xi^{\text {set }}=B^{\dagger} P^{\text {set }}=$ $P_{I}^{\text {set }}$. For general acyclic graphs, the mapping between nodal power injections and branch flows is one-to-one. As a consequence, the set of designable branch flows is exactly the image under $B^{\dagger}$ of the set of $\gamma$-feasible power injection set-points, and no larger.

\section{EXTENSIONS OF SECONDARY CONTROL}

\section{A. Distributed-Averaging PI-control by Shafiee et al.}

Another secondary distributed PI controller has been presented in [14]. The proposed secondary-control input $u_{i}(t)$ to the dynamics (3)-(4) is given by an integral feedback ${ }^{\ddagger}$ of the weighted average frequency ${ }^{\S}$ among the inverters:

$$
u_{i}(t)=-p_{i}, \quad k_{i} \dot{p}_{i}=\frac{\sum_{j \in \mathcal{V}_{I}} D_{j} \dot{\theta}_{j}}{\sum_{j \in \mathcal{V}_{I}} D_{j}}
$$

Here, $p_{i} \in \mathbb{R}$ is again an auxiliary power variable and $k_{i}>0$ is a gain, for each $i \in \mathcal{V}_{I}$. By explicit numerical counter-examples for non-symmetric setups of inverters (e.g.,

\footnotetext{
¥The controller proposed in [14] also contains a proportional feedback of the average frequency. We found that such a proportional feedback destroys the desired proportional power sharing, unless the gains are carefully tuned. For these reasons and since the resulting closed loop is hardly amenable to an analytic investigation, we omit the proportional feedback channel here.

$\S^{\S}$ The controller in [14] contains a true arithmetic average with all $D_{i}=1$ in (12). Since the synchronization frequency (5) is obtained by a weighted average, we found the choice (12) more appealing and intuitive. Simulation studies suggest that any convex combination of the inverter frequencies yields identical results.
}

non-identical inverters and non-uniform line admittances in a parallel topology), it can be shown that the closed-loop system (3)-(4) with (12) fails to achieve power sharing for arbitrary values of $k_{i}>0$. Hence, the values of $k_{i}$ need to be carefully chosen. In the following, we suggest the choice

$$
k_{i}=k / D_{i}, \quad i \in \mathcal{V}_{I} .
$$

where $k>0$ is constant. That is, the integral channels have the time-constants inverse to the proportional droop control channels (4). In this case, the closed loop is given by

$$
\begin{aligned}
0 & =P_{i}^{*}-P_{\mathrm{e}, i}(\theta), & & i \in \mathcal{V}_{L}, \\
D_{i} \dot{\theta}_{i} & =P_{i}^{*}-P_{\mathrm{e}, i}(\theta)-p_{i}, & & i \in \mathcal{V}_{I}, \\
k \frac{\dot{p}_{i}}{D_{i}} & =\frac{\sum_{j \in \mathcal{V}_{I}} D_{j} \dot{\theta}_{j}}{\sum_{j \in \mathcal{V}_{I}} D_{j}}, & & i \in \mathcal{V}_{I} .
\end{aligned}
$$

By changing coordinates $q_{i}=p_{i} / D_{i}-\omega_{\text {avg }}$ for $i \in \mathcal{V}_{I}$ and observing that $k \dot{q}_{i}=\frac{\sum_{j \in \mathcal{V}_{I} D_{j} \dot{\theta}_{j}}}{\sum_{j \in \mathcal{V}_{I}} D_{j}}$ is identical for all $i \in \mathcal{V}_{I}$, we can rewrite the closed-loop equations (14) as

$$
\begin{aligned}
0 & =\widetilde{P}_{i}-P_{\mathrm{e}, i}(\theta), & i \in \mathcal{V}_{L}, \\
D_{i} \dot{\theta}_{i} & =\widetilde{P}_{i}-P_{\mathrm{e}, i}(\theta)-D_{i} q, & i \in \mathcal{V}_{I}, \\
k \dot{q} & =\frac{\sum_{j \in \mathcal{V}_{I}} D_{j} \dot{\theta}_{j}}{\sum_{j \in \mathcal{V}_{I}} D_{j}} . &
\end{aligned}
$$

Notice that equation (15c) in the transformed system can be implemented as a centralized integrator. For these reasons, we refer to the controller (4), (12) with the choice of gains (13) as the centralized-averaging proportional- integral (CAPI) controller. This perspective is not only insightful and shows the communication complexity of the CAPI controller (12)-(13), but equations (15) are also convenient for a stability analysis resulting in the following theorem.

Theorem 4.1: (Stability of CAPI-Controlled Network). Consider an acyclic network of droop-controlled inverters and loads in which all inverters can communicate and average their frequencies, as described by the closed-loop system (14) with parameters $k>0, P_{i}^{*} \in\left[0, \bar{P}_{i}\right]$, and $D_{i}>0$ for $i \in \mathcal{V}_{I}$. The following two statements are equivalent:

(i) Stability of Droop Controller: The droop control stability condition (8) holds;

(ii) Stability of CAPI Controller: There exists an arc length $\gamma \in[0, \pi / 2[$ such that the system (14) possesses a locally exponentially stable and unique ${ }^{\dagger}$ equilibrium $\left(\theta^{*}, p^{*}\right) \in \Delta_{G}(\gamma) \times \mathbb{R}^{n_{I}}$.

If the equivalent statements (i) and (ii) hold true, then the unique equilibrium is given as in Theorem 2.1 (ii), along with $p_{i}^{*}=D_{i} \omega_{\text {avg }}$ for $i \in \mathcal{V}_{I}$. Moreover, if the droop coefficients are selected proportionally, then the CAPI controller (12)(13) preserves the proportional power sharing property of the primary droop controller (4).

Proof: We start by writing the closed loop (15) in vector form analogous to [10]. Let $D_{I}=\operatorname{diag}\left(\left\{D_{i}\right\}_{i \in \mathcal{V}_{I}}\right)$, and let $D_{\text {tot }}=\sum_{i \in \mathcal{V}_{I}} D_{i}=\mathbf{1}^{T} D_{I} \mathbf{1}$. Let $\widetilde{P}=\left(\widetilde{P}_{L}^{T}, \widetilde{P}_{I}^{T}\right)^{T}$, and accordingly let $P_{e}(\theta)=\left(P_{e, L}(\theta)^{T}, P_{e, I}(\theta)^{T}\right)^{T}$, where

\footnotetext{
${ }^{\dagger}$ Modulo the rotational symmetry inherent in the model.
} 
$P_{e, I}(\theta)$ and $P_{e, L}(\theta)$ are vectors of power injections (2) at the inverters $\mathcal{V}_{I}$ and the loads $\mathcal{V}_{L}$. Let the angles be partitioned accordingly as $\theta=\left(\theta_{L}, \theta_{I}\right)$. With this notation, the closedloop dynamics (15) read in vector form as

$$
\begin{aligned}
& \underbrace{\left[\begin{array}{ccc}
I & \mathbf{0} & \mathbf{0} \\
\mathbf{0} & D_{I} & \mathbf{0} \\
\mathbf{0} & \mathbf{0} & k \cdot D_{\mathrm{tot}}
\end{array}\right]}_{\triangleq Q_{1}}\left[\begin{array}{c}
\mathbf{0} \\
\dot{\theta}_{I} \\
\dot{q}
\end{array}\right]=\left[\begin{array}{ccc}
I & \mathbf{0} & \mathbf{0} \\
\mathbf{0} & I & D_{I} \mathbf{1} \\
\mathbf{0} & \mathbf{1}^{T} & D_{\mathrm{tot}}
\end{array}\right]\left[\begin{array}{c}
\widetilde{P}_{L}-P_{e, L}(\theta) \\
\widetilde{P}_{I}-P_{e, I}(\theta) \\
-q
\end{array}\right] \\
& =\underbrace{\left[\begin{array}{ccc}
I & \mathbf{0} & \mathbf{0} \\
\mathbf{0} & D_{I} & \mathbf{0} \\
\mathbf{0} & \mathbf{0} & 1
\end{array}\right]}_{\triangleq Q_{2}} \underbrace{\left[\begin{array}{ccc}
I & \mathbf{0} & \mathbf{0} \\
\mathbf{0} & D_{I}^{-1} & \mathbf{1} \\
\mathbf{0} & \mathbf{1}^{T} & D_{\mathrm{tot}}
\end{array}\right]}_{\triangleq Q_{3}} \underbrace{\left[\begin{array}{c}
\widetilde{P}_{L}-P_{e, L}(\theta) \\
\widetilde{P}_{I}-P_{e, I}(\theta) \\
-q
\end{array}\right]}_{\triangleq x} .
\end{aligned}
$$

The matrices $Q_{1}$ and $Q_{2}$ are nonsingular. The matrix $Q_{3}$ is singular with $\operatorname{ker}\left(Q_{3}\right)=\left[0\left(D_{I} \mathbf{1}\right)^{T}-1\right]^{T}$ corresponding to decreasing the secondary variable $q$ and increasing all inverter flows accordingly. On the other hand, we have that $\left[\begin{array}{lll}\mathbf{1}^{T} & \mathbf{1}^{T} & 0\end{array}\right] x=0$ due to balanced injections $\mathbf{1}^{T} \widetilde{P}=0$ and symmetry of the flow $\mathbf{1}^{T} P_{e}(\theta)=0$. We conclude that $x \notin \operatorname{ker}\left(Q_{3}\right)$. Thus, possible equilibria of (16) are given by $x=0$, that is, the set of desired equilibria $\theta^{*}$ from (7) and $q^{*}=0$. Equivalently, from Theorem 2.1, the equation $x=0$ is solvable for a unique (modulo rotational symmetry) value $\theta^{*} \in \Delta_{G}(\gamma)$ if and only if the parametric condition (8) holds.

To establish stability of the equilibrium $\left(\theta^{*}, 0\right)$, we follow the proof strategy of [10, Theorem 8$]$. Recall that the negative load flow Jacobian $-\partial / \partial \theta\left(\widetilde{P}-P_{e}(\theta)\right)$ is given by

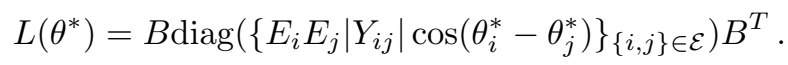

For $\theta^{*} \in \Delta_{G}(\gamma), \gamma \in\left[0, \pi / 2\left[\right.\right.$, we have $\cos \left(\theta_{i}^{*}-\theta_{j}^{*}\right) \geq$ $\cos (\gamma)>0$. Consequently, $L\left(\theta^{*}\right)$ is a positive semidefinite Laplacian matrix, see [18, Lemma 2]. Thus, the linearization of the DAE (16) about the regular fixed point $\left(\theta^{*}, 0\right)$ and elimination of the algebraic equations results in the Jacobian

$$
J\left(\theta^{*}\right)=\underbrace{\left[\begin{array}{cc}
I & \mathbf{0} \\
\mathbf{0} & \left(k \cdot D_{\mathrm{tot}}\right)^{-1}
\end{array}\right]}_{\triangleq \widetilde{Q}_{1}} \underbrace{\left[\begin{array}{cc}
D_{I}^{-1} & \mathbf{1} \\
\mathbf{1}^{T} & D_{\mathrm{tot}}
\end{array}\right]}_{\triangleq \widetilde{Q}_{2}} \underbrace{\left[\begin{array}{cc}
-L_{\mathrm{red}}\left(\theta^{*}\right) & \mathbf{0} \\
\mathbf{0} & -1
\end{array}\right]}_{\triangleq X},
$$

where $L_{\mathrm{red}}\left(\theta^{*}\right)$ is the Schur complement of $L\left(\theta^{*}\right)$ with respect to the entries corresponding to the loads $\mathcal{V}_{I}$. It is known that $L_{\text {red }}\left(\theta^{*}\right)$ is again a positive semidefinite Laplacian matrix [19, Lemma II.1]. The matrix $\widetilde{Q}_{1}$ is diagonal and positive definite, and $\widetilde{Q}_{2}$ is positive semidefinite with $\operatorname{ker}\left(\widetilde{Q}_{2}\right)=\left[\left(D_{I} \mathbf{1}\right)^{T}-1\right]^{T}$. We will proceed via a continuitytype argument. Consider momentarily the perturbed Jacobian $J_{\epsilon}\left(\theta^{*}\right)$, where $\widetilde{Q}_{2}$ is replaced by the positive definite matrix $\widetilde{Q}_{2, \epsilon}=\left[\begin{array}{cc}D_{I}^{-1} & 1 \\ \mathbf{1}^{T} & D_{\mathrm{tot}}+\epsilon\end{array}\right]$, where $\epsilon>0$. The eigenvalues of $J_{\epsilon}\left(\theta^{*}\right)$ are obtained from $\widetilde{Q}_{1} \widetilde{Q}_{2, \epsilon} X v=\lambda v$ for some $(\lambda, v) \in$ $\mathbb{C} \times \mathbb{C}^{n_{I}+1}$. Equivalently, let $y=\widetilde{Q}_{1}^{-1} v$, then we obtain

$$
-\widetilde{Q}_{2, \epsilon} \cdot \operatorname{blkdiag}\left(L_{\mathrm{red}}, 1 /\left(k \cdot D_{\mathrm{tot}}\right)\right) y=\lambda y
$$

By applying the Courant-Fischer Theorem to this generalized eigenvalue problem, we conclude, for $\epsilon>0$ and modulo rotational symmetry, all eigenvalues $\lambda$ are real and negative.
Now, consider again the unperturbed case with $\epsilon=0$. Recall that $\operatorname{ker}\left(\widetilde{Q}_{2}\right)=\left[\left(D_{I} \mathbf{1}\right)^{T}-1\right]^{T}$, and the image of the matrix $\operatorname{blkdiag}\left(L_{\text {red }}, 1 /\left(k \cdot D_{\text {tot }}\right)\right)$ excludes $\operatorname{span}\left(\left[\begin{array}{ll}\mathbf{1}^{T} & 0\end{array}\right]^{T}\right)$. It follows that $\widetilde{Q}_{2, \epsilon} \cdot \operatorname{blkdiag}\left(L_{\text {red }}, 1 /\left(k \cdot D_{\text {tot }}\right)\right) y$ is zero if only if $y \in \operatorname{span}\left(\left[\mathbf{1}^{T} 0\right]^{T}\right)$ corresponding to the rotational symmetry. We conclude that the number of negative real eigenvalues of $J_{\epsilon}\left(\theta^{*}\right)$ does not change as $\epsilon \searrow 0$. Hence, the equilibrium equilibrium $\left(\theta^{*}, 0\right)$ of the DAE (16) is (again, modulo rotational symmetry) locally exponentially stable.

\section{B. Extension of secondary control to lossy networks}

We now study the operation of the DAPI controller when the network contains transfer conductances - that is, unlike in Section II, the transmission lines are no longer assumed to be purely inductive. The admittance matrix $Y \in \mathbb{C}^{n \times n}$ is now given by $Y=G_{i j}+\sqrt{-1} T_{i j}$, where $G \in \mathbb{R}^{n \times n}$ is the conductance matrix and $T \in \mathbb{R}^{n \times n}$ is the susceptance matrix. The conductances are responsible for power losses in the network. The active electrical power $P_{\mathrm{e}, i} \in \mathbb{R}$ injected into the network at node $i \in\{1, \ldots, n\}$ is then given by [17]

$$
P_{\mathrm{e}, i}(\theta)=\sum_{j=1}^{n} E_{i} E_{j}\left|Y_{i j}\right| \sin \left(\theta_{i}-\theta_{j}-\phi_{i j}\right)
$$

where $\left|Y_{i j}\right|^{2}=G_{i j}^{2}+T_{i j}^{2}$ and $\phi_{i j} \triangleq-\arctan \left(G_{i j} / T_{i j}\right)$. In vector notation, the frequency droop controller is then

$$
D \dot{\theta}=P^{*}-P_{e}(\theta),
$$

where $D=\operatorname{diag}\left(\mathbf{0}_{\left|\mathcal{V}_{L}\right|},\left\{D_{i}\right\}_{i \in \mathcal{V}_{I}}\right)$. The question of existence and uniqueness of stable equilibria for the system (17), (18) is considerably more challenging than in the lossless case. Due to the lack of simple and sharp existence and uniqueness conditions for lossy networks, we make use of assumptions concerning the primary-controlled system (17), (18) and use these to study the secondary-controlled systems, DAPI control (10), (17) and CAPI control (14), (17). The first result shows that both controllers succeed in eliminating deviations from the rated frequency, assuming they lead to a synchronized steady state.

Lemma 4.2: (Synchronization Frequency is Zero). If the frequencies of the inverters in the DAPI-controlled system (10), (17) and in the CAPI-controlled system (14), (17) are synchronized, and if the auxiliary power variables $p$ are in steady state, then the synchronization frequency is zero.

Proof: Assume there exist two solutions $(\bar{\theta}, \bar{p}) \in$ $\Delta_{G}(\gamma) \times \mathbb{R}^{n_{I}}$ and $(\widetilde{\theta}, \widetilde{p}) \in \Delta_{G}(\gamma) \times \mathbb{R}$ for the systems $(10)$, (17) and (14), (17), respectively, such that $\dot{\bar{\theta}}=\bar{\omega}_{\text {sync }} \mathbf{1} \in$ $\mathbb{R}^{n_{I}}, \bar{p}=\operatorname{const} \mathbf{1}, \dot{\tilde{\theta}}=\widetilde{\omega}_{\text {sync }} \mathbf{1} \in \mathbb{R}^{n_{I}}$, and $\widetilde{p}=\operatorname{const} \mathbf{1}$.

By summing up the equations (10c) in steady state, we get $0=\sum_{i \in \mathcal{V}_{I}} k_{i} \dot{\bar{p}}_{i}=\sum_{i \in \mathcal{V}_{I}} D_{i} \bar{\omega}_{\text {sync }}$. Since $D_{i}>0$ for all $i \in \mathcal{V}_{I}, \bar{\omega}_{\text {sync }}$ is zero .

For the CAPI, we obtain in steady state $0=k_{i} \dot{\widetilde{p}}_{i}=$ $\frac{\sum_{j \in \mathcal{V}_{I} D_{j}}}{\sum_{j \in \mathcal{V}_{I}} D_{j}} \widetilde{\omega}_{\text {sync }}$ for $i \in \mathcal{V}_{I}$. Thus, $\widetilde{\omega}_{\text {sync }}$ is zero.

We will now characterize possible solutions of the DAPIcontrolled system (10), (17) and the CAPI-controlled system (14), (17). First, due to the lack of general parametric conditions, we assume the existence of a solution to the frequency-droop-controlled system (17), (18). 
Note that in the following results, all uniqueness properties are modulo the rotational symmetry inherent in the model.

Assumption 2: (Existence and uniqueness for the frequency-droop control system with losses). There exists an arc length $\gamma \in[0, \pi / 2[$ such that the closed-loop system (18) possesses a unique and frequency-synchronized solution $t \mapsto \theta^{*}(t)=\theta_{0}^{*}+\left(\omega_{\text {sync }}^{*} t \mathbf{1}_{n}\right)(\bmod 2 \pi) \in \Delta_{G}(\gamma)$, with $\omega_{\text {sync }}^{*} \in \mathbb{R}$ for all $t \geq 0$.

By summing over the equations (18) in steady state, we obtain the corresponding unique synchronization frequency

$$
\omega_{\mathrm{sync}}^{*}=\frac{\sum_{i \in \mathcal{V}} P_{i}^{*}-\sum_{i \in \mathcal{V}} P_{\mathrm{e}, i}\left(\theta^{*}\right)}{\sum_{i \in \mathcal{V}_{I}} D_{i}} .
$$

If Assumption 2 holds, then $\theta^{*}$ is the unique solution in $\Delta_{G}(\gamma)$ of the algebraic equation

$$
P^{*}-P_{e}(\theta)-D \frac{\sum_{i \in \mathcal{V}} P_{i}^{*}-\sum_{i \in \mathcal{V}} P_{\mathrm{e}, i}(\theta)}{\sum_{i \in \mathcal{V}_{I}} D_{i}} \mathbf{1}=\mathbf{0}
$$

Theorem 4.3: (Existence and Uniqueness of Sync'd Solutions for DAPI-/CAPI- Controlled systems in Lossy Networks). If Assumption 2 holds, then the unique synchronized solutions for the DAPI-controlled system (10), (17) and the CAPI-controlled system (14), (17) are given by the pair $(\theta, p)=\left(\theta^{*}, D_{I} \omega_{\text {sync }}^{*} \mathbf{1}\right)$.

Proof: We have proven in Lemma 4.2 that the only possible synchronization frequency is 0 . Therefore, we only look for equilibria of the systems (10), (17) and (14), (17). First consider the DAPI controller (10), (17). The equilibria of system (10), (17) have to satisfy the equations

$$
\begin{aligned}
& \mathbf{0}=P^{*}-P_{e}(\theta)-\left[\begin{array}{c}
\mathbf{0}_{\left|\mathcal{V}_{L}\right|} \\
p
\end{array}\right], \\
& \mathbf{0}=-L_{c} D_{I}^{-1} p .
\end{aligned}
$$

In order to satisfy (20b), $D_{I}^{-1} p$ must be in the kernel of $L_{c}$ and thus have the form $c \mathbf{1}$ with $c \in \mathbb{R}$. Therefore, every $p$ must have the form $c D_{I} \mathbf{1}$. Suppose that $(\theta, p)=$ $\left(\bar{\theta}, \bar{\omega}_{\text {sync }} D_{I} \mathbf{1}\right) \in \Delta_{G}(\gamma) \times \mathbb{R}^{n_{I}}$ satisfies equations (20). We have

$$
\mathbf{0}=P^{*}-P_{e}(\bar{\theta})-\bar{\omega}_{\text {sync }} D \mathbf{1}
$$

By summing over these equations we get

$$
\bar{\omega}_{\text {sync }}=\frac{\sum_{i \in \mathcal{V}} P^{*}-\sum_{i \in \mathcal{V}} P_{e}(\bar{\theta})}{\sum_{i \in \mathcal{V}} D_{i}} .
$$

By inserting (22) in equation (21), we obtain

$$
\mathbf{0}=P^{*}-P_{e}(\bar{\theta})-D \frac{\sum_{i \in \mathcal{V}} P^{*}-\sum_{i \in \mathcal{V}} P_{e}(\bar{\theta})}{\sum_{i \in \mathcal{V}} D_{i}} \mathbf{1}
$$

which is simply (19). Assumption 2 states that $\theta^{*}$ is the only solution in $\Delta_{G}(\gamma)$ of (19). Therefore, $\bar{\theta}=\theta^{*}$ and $\bar{\omega}_{\text {sync }}=$ $\omega_{\text {sync }}^{*}$. It can be verified that the pair $\left(\theta^{*}, \omega_{\text {sync }}^{*} D_{I} \mathbf{1}\right)$ is an actual equilibrium of (20).

Now, consider the CAPI controller in its transformed coordinates (15), (17). The equilibria of (15), (17) satisfy

$$
\mathbf{0}=P^{*}-D \omega_{\text {sync }}^{*} \mathbf{1}-P_{e}(\theta)-D q \mathbf{1} .
$$

Suppose that $(\theta, q)=\left(\widetilde{\theta}, \widetilde{\omega}_{\text {sync }}\right) \in \Delta_{G}(\gamma) \times \mathbb{R}$ satisfies equations (23). Thus, this pair satisfies the equations

$$
\mathbf{0}=P^{*}-P_{e}(\widetilde{\theta})-\left(\widetilde{\omega}_{\text {sync }}+\omega_{\text {sync }}^{*}\right) D \mathbf{1} .
$$

Note that (24) is the same as (21). By analogous reasoning, we obtain that $\widetilde{\theta}=\theta$ and $\widetilde{\omega}_{\text {sync }}+\omega_{\text {sync }}^{*}=\omega_{\text {sync }}^{*}$. A transformation back to the original coordinates leads to the pair $\left(\theta^{*}, D_{I} \omega_{\text {sync }}^{*} 1\right)$ as the unique equilibrium of (23).

Having established the existence of equilibria for the secondary-controlled systems, we now study their stability properties. Simulations of a network of parallel inverters suggest that exponential stability is maintained independent of the conductance magnitudes. While it is difficult to find a general analytic proof for the stability of the equilibria independent of the conductance magnitudes, we can extend the stability from the lossless case using a continuity-type argument for the eigenvalues of the Jacobian.

Theorem 4.4: (Stability of Equilibria for DAPI/CAPI Controllers in Lossy Networks). Let Assumption 2 hold and let $\left(\theta^{*}, D_{I} \omega_{\text {sync }}^{*} \mathbf{1}\right) \in \bar{\Delta}_{G}(\gamma) \times \mathbb{R}^{n_{I}}$ be the unique synchronized solution of the DAPI-controlled system (10), (17) and of the CAPI-controlled system (14), (17) with $\gamma \in[0, \pi / 2[$. There exists a $\varepsilon>0$ such that if $\|G\|<\varepsilon$, the equilibrium given by $\left(\theta^{*}, D_{I} \omega_{\text {sync }}^{*} \mathbf{1}\right)$ is locally exponentially stable for both systems (10), (17) and (14), (17).

Proof: The lossy DAPI system (10), (17) is a smooth perturbation of the nominal lossless DAPI system (10), (2). Recall that the eigenvalues of the Jacobian, resulting from the linearization of DAE (10) around $\left(\theta^{*}, D_{I} \omega_{\text {sync }}^{*} \mathbf{1}\right)$ and the elimination of the algebraic equations, are continuous function of the conductances $G_{i j}$. Notice that conductances do not affect the rotational symmetry, and the Jacobian maintains its zero eigenvalue and associated eigenvector. Since the remaining eigenvalues of the nominal system are in the open left half plane, then - due to continuity and for sufficiently small conductances - the nonzero eigenvalues of the lossy system are also in the open left half plane.

It turns out that the proportional power sharing properties of the closed loop are maintained in the presence of losses for both the primary- and the secondary-controlled systems.

Theorem 4.5: (Power Sharing in Lossy Networks). Let Assumption 2 hold and consider a network of droopcontrolled inverters and loads as described by (18).

(i) If we select the droop coefficients and the set points of the inverters proportionally, meaning that the ratios $D_{i} / P_{i}^{*}$ and $P_{i}^{*} / \bar{P}_{i}$ are constant for all $i \in \mathcal{V}$, then the inverters share the load $P_{L}$ proportionally according to their power ratings, that is, $P_{\mathrm{e}, i} / \bar{P}_{i}=P_{e, j} / \bar{P}_{j}$, for each $i \in \mathcal{V}$.

(ii) The secondary controllers DAPI and CAPI, proposed in (9) and (12), respectively, preserve power sharing.

Proof: The proof for partial DAPI control (resp. partial CAPI control) is identical to the proof in the lossless case of [10, Theorem 7] (resp. Theorem 4.1).

\section{Partial DAPI/CAPI Control}

The DAPI controller (9) requires a connected communication network among the inverters. Due to extreme distance 


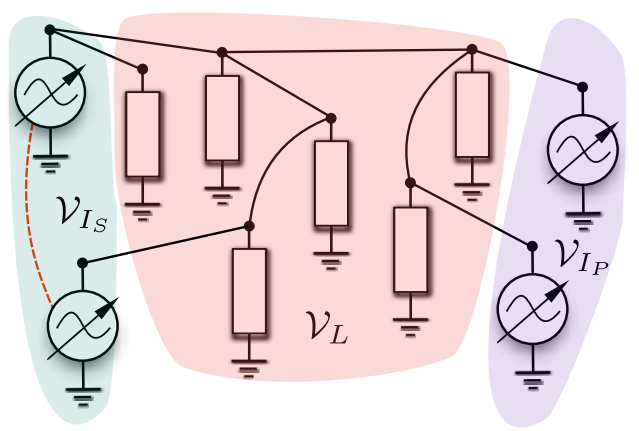

Fig. 1. Schematic of partial secondary control. The red dotted line represents a communication link.

or other barriers, it may be desirable to have only a subset of inverters within the network assist in regulating the network frequency (see Figure 1).

To investigate this scenario, we partition the set of inverters as $\mathcal{V}_{I_{P}} \cup \mathcal{V}_{I_{S}}=\mathcal{V}_{I}$, where the action of the $\mathcal{V}_{I_{P}}$ inverters is restricted to primary droop control, and the $\mathcal{V}_{I_{S}}$ inverters perform the secondary DAPI/CAPI control. Under this partitioning of nodes, the inverter control equations become

$$
\begin{aligned}
D_{i} \dot{\theta}_{i} & =P_{i}^{*}-P_{\mathrm{e}, i}(\theta), & & i \in \mathcal{V}_{I_{P}}, \\
D_{i} \dot{\theta}_{i} & =P_{i}^{*}-P_{\mathrm{e}, i}(\theta)+u_{i}(t), & & i \in \mathcal{V}_{I_{S}},
\end{aligned}
$$

The scaled power imbalance is now defined by

$$
\omega_{\text {pavg }} \triangleq \sum_{j \in \mathcal{V}} P_{i}^{*} /\left(\sum_{j \in \mathcal{V}_{I_{S}}} D_{i}\right)
$$

Theorem 4.6: (Stability of Partial secondary-controlled Network). Consider an acyclic network of droop-controlled inverters and loads, as described by the closed-loop system (3), (25), with parameters $P_{i}^{*} \in\left[0, \bar{P}_{i}\right]$ and $D_{i}>0$ for $i \in \mathcal{V}_{I}$. The secondary-control input $u_{i}$ is defined by either (9) (DAPI) or (12) (CAPI) for all $i \in \mathcal{V}_{I_{S}}$ with $k_{i}>0$. The following two statements are equivalent:

(i) Stability of Droop Controller: The droop control stability condition (8) holds;

(ii) Stability of the Secondary Controllers: There exists an arc length $\gamma \in[0, \pi / 2[$ such that the two systems (3), (25), (9) and (3), (25), (12) possess the same locally exponentially stable and unique equilibrium $\left(\theta^{*}, p^{*}\right) \in \Delta_{G}(\gamma) \times \mathbb{R}^{\left|\mathcal{V}_{I_{S}}\right|}$.

If the equivalent statements (i) and (ii) hold true, then the unique equilibrium is given as in Theorem 2.1 (ii), with $\omega_{\text {avg }}$ given by (26) along with $p_{i}^{*}=D_{i} \omega_{\text {pavg }}$ for $i \in \mathcal{V}_{I_{S}}$. The synchronized solution satisfies $\theta^{*}(t)=\theta_{0}+\left(\omega_{\text {sync }} t \mathbf{1}_{n}\right)$ $(\bmod 2 \pi)$ for some $\theta_{0} \in \Delta_{G}(\gamma)$, where $\omega_{\text {sync }}=\omega_{\text {avg }}$,

Proof: The proof for partial DAPI control (resp. partial CAPI control) is analogous to the proof of [10, Theorem 8] (resp. Theorem 4.1), while carefully accounting for the partition $\mathcal{V}_{I}=\mathcal{V}_{I_{P}} \cup \mathcal{V}_{I_{S}}$ in the Jacobian matrices.

Theorem 4.6 shows that partial secondary control strategies are able to successfully stabilize the network. We now investigate the power sharing properties of the partial
DAPI/CAPI control schemes. The steady-state power injection at the equilibrium $\left(\theta^{*}, p^{*}\right)$ is

$$
\begin{aligned}
P_{\mathrm{e}, i}\left(\theta^{*}\right) & =P_{i}^{*}, & i \in \mathcal{V}_{I_{P}} \cup \mathcal{V}_{L}, \\
P_{\mathrm{e}, i}\left(\theta^{*}\right) & =P_{i}^{*}-D_{i} \omega_{\mathrm{sync}}, & i \in \mathcal{V}_{I_{S}} .
\end{aligned}
$$

From the first of these equations, we see that inverters in $\mathcal{V}_{I_{P}}$ are effectively negative loads. Let $\widetilde{\mathcal{V}}_{L}=\mathcal{V}_{I_{P}} \cup \mathcal{V}_{L}$ be the set of loads in steady state and $\widetilde{\mathcal{V}}_{I}=\mathcal{V}_{I_{S}}$ be the set of inverters. Applying [10, Theorem 7] to the modified sets $\widetilde{\mathcal{V}}_{I}$ and $\widetilde{\mathcal{V}}_{L}$ gives the following result.

Theorem 4.7: (Power Flow Constraints and Power Sharing). Consider the same setup as in Theorem 4.6, and define the total load by $P_{L} \triangleq \sum_{i \in \mathcal{V}_{L}} P_{i}^{*}$. If the droop coefficients and the set points of the inverters that perform secondary control are selected proportionally, meaning that the ratios $D_{i} / P_{i}^{*}$ and $P_{i}^{*} / \bar{P}_{i}$ are constant for all $i \in \mathcal{V}_{I_{S}}$, then the following two statements are equivalent:

(i) Injection Constraints: $0 \leq P_{e, i}\left(\theta^{*}\right) \leq \bar{P}_{i} \quad \forall i \in \mathcal{V}_{I}$.

(ii) Load and Set Point Constraints:

$$
\begin{aligned}
& \sum_{j \in \mathcal{V}_{I_{P}}} P_{j}^{*} \leq-P_{L} \leq \sum_{j \in \mathcal{V}_{I_{P}}} P_{j}^{*}+\sum_{j \in \mathcal{V}_{I_{S}}} \bar{P}_{j}, \\
& 0 \leq P_{i}^{*} \leq \bar{P}_{i} \quad \forall i \in \mathcal{V}_{I_{P}} .
\end{aligned}
$$

Moreover, the inverters performing secondary control share the load residual $P_{L}-\sum_{i \in \mathcal{V}_{I_{P}}} P_{i}^{*}$ proportionally according to their power ratings, i.e., $P_{\mathrm{e}, i} / \bar{P}_{i}=P_{e, j} / \bar{P}_{j}$ for all $i \in \mathcal{V}_{I_{S}}$.

\section{Simulation Study}

We illustrate a subset of our results by means of a simulation where two inverters operating in parallel supply a variable load. Since the DAPI controller (9) is thoroughly covered in [10], we show the robustness and transient performance of the CAPI controller (12)-(13) originally proposed in [14]. The voltage magnitude at each inverter is controlled via the quadratic voltage-droop method [20]

$$
\tau_{i} \dot{E}_{i}=-C_{i} E_{i}\left(E_{i}-E_{i}^{*}\right)-Q_{i}, \quad i \in \mathcal{V}_{I},, \quad i \in\{1,2\},
$$

where $E_{i}^{*}>0$ is the nominal voltage magnitude, $C_{i}>0$ (resp. $\tau_{i}>0$ ) is the proportional (resp. integral) quadratic voltage-droop coefficient, and $Q_{\mathrm{e}, i} \in \mathbb{R}$ is the reactive power injection [17]. The simulation parameters are reported in Table I, and a time-domain simulation is shown in Figure 2.

TABLE I

PARAMETER VALUES FOR SIMULATION IN FIGURE 2.

\begin{tabular}{llr}
\hline Parameter & Symbol & Value \\
\hline Nom. Frequency & $\omega^{*} / 2 \pi$ & $60 \mathrm{~Hz}$ \\
Nom. Voltages & $E_{i}^{*}$ & {$[120,122] \mathrm{V}$} \\
Output/Line Induc. & $L_{i}$ & {$[0.7,0.5] \mathrm{mH}$} \\
Output/Line Resist. & $R_{i}$ & {$[0.14,0.1] \Omega$} \\
Inv. Ratings $(P)$ & $P_{i}^{*}=\bar{P}_{i}$ & {$[2,3] \mathrm{kW}$} \\
Load $(P)$ & $P_{0}^{*}(t)$ & $P_{0}^{*} \in\{-2.5,-5\} \mathrm{kW}$ \\
Load $(Q)$ & $Q_{0}^{*}(t)$ & $Q_{0}^{*} \in\{-.5,-1\} \mathrm{kvar}$ \\
$\omega-$ Droop Coeff. & $D_{i}$ & {$[4,6] \times 10^{-4} \mathrm{~W} \cdot \mathrm{s}$} \\
Sec. Droop Coeff. & $k_{i}$ & $10^{-9} \mathrm{~s}$ \\
Quadratic E-Droop Coeff. & $C_{i}$ & {$[1,1.5] 10^{-3} \mathrm{~s}$} \\
Quadratic $E$-Droop Int. Coeff. & $\tau_{i}$ & {$[5,5] \mathrm{s}$} \\
\hline
\end{tabular}

Observe that the CAPI controlled system (14) is robust and achieves an acceptable transient performance in presence 

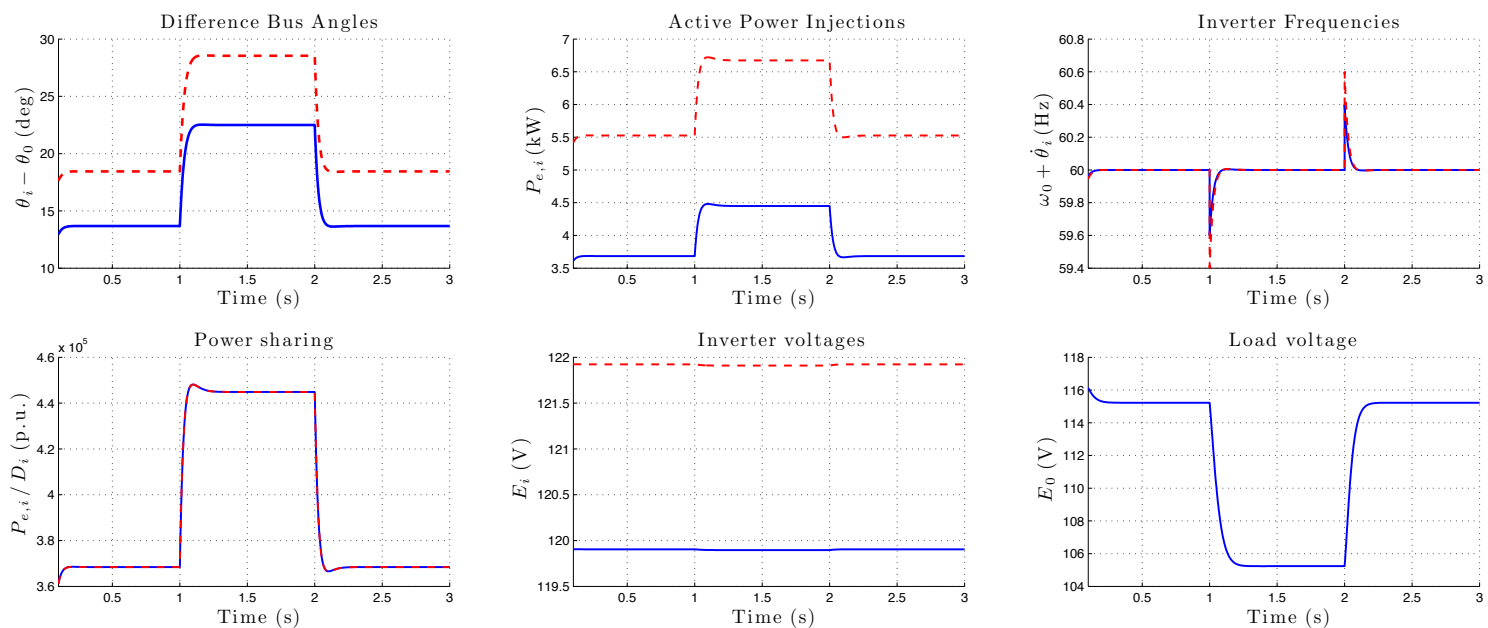

Fig. 2. CAPI-controlled closed loop (14) for two inverters supplying a load. The solid (blue) plots correspond to inverter 1, and the dashed (red) plots correspond to inverter 2 . The simulation is initialized at a non-stationary value, and the active and reactive power demand at the load doubles at $t=1 \mathrm{~s}$ and then returns to its original vaue at $t=2 \mathrm{~s}$.

of lossy lines and unmodeled reactive power and voltage dynamics. Note that the inverse droop coefficients $D_{i}$ must be chosen extremely small* to achieve an acceptable performance for the frequency dynamics. However, this choice also results in an overly aggressive primary droop controller (4). If the coefficients $D_{i}$ are selected within a more reasonable range, then the CAPI integral control is slow and generally ineffective. Whereas our choice of coefficients in Table I is certainly not optimal, it illustrates a disadvantage of CAPI controller (12)-(13) over the DAPI controller (9) (besides communication complexity): the time constants of the proportional feedback (4) and the integral feedback (12) are coupled through (13) and cannot be chosen independently if one wishes to achieve power sharing.

\section{CONCLUSIONS}

In this work we have presented several extensions of primary and secondary control in microgrids. In particular, In Subsection IV-B we have shown that if the primary controller succeeds in stabilizing the network, then the DAPI/CAPI secondary controllers effectively regulate the frequency, even in the presence of losses. Removing the assumption on small conductances and subsequently proving exponential stability is a challenging and open problem.

\section{REFERENCES}

[1] Q.-C. Zhong and T. Hornik, Control of Power Inverters in Renewable Energy and Smart Grid Integration. Wiley-IEEE Press, 2013.

[2] J. M. Guerrero, J. C. Vasquez, J. Matas, L. G. de Vicuna, and M. Castilla, "Hierarchical control of droop-controlled AC and DC microgrids-a general approach toward standardization," IEEE Transactions on Industrial Electronics, vol. 58, no. 1, pp. 158-172, 2011.

[3] J. A. P. Lopes, C. L. Moreira, and A. G. Madureira, "Defining control strategies for microgrids islanded operation," IEEE Transactions on Power Systems, vol. 21, no. 2, pp. 916-924, 2006.

[4] M. C. Chandorkar, D. M. Divan, and R. Adapa, "Control of parallel connected inverters in standalone AC supply systems," IEEE Transactions on Industry Applications, vol. 29, no. 1, pp. 136-143, 1993.

*The DAPI control scheme in $[10]$ uses $\left[D_{1}, D_{2}\right]=[4,6] \times 10^{3} \mathrm{~W} \cdot \mathrm{s}$.
[5] A. Tuladhar, H. Jin, T. Unger, and K. Mauch, "Parallel operation of single phase inverter modules with no control interconnections," in Applied Power Electronics Conference and Exposition, Atlanta, GA, USA, Feb. 1997, pp. 94-100.

[6] S. Barsali, M. Ceraolo, P. Pelacchi, and D. Poli, "Control techniques of dispersed generators to improve the continuity of electricity supply," in IEEE Power Engineering Society Winter Meeting, New York, NY, USA, Jan. 2002, pp. 789-794.

[7] R. Majumder, A. Ghosh, G. Ledwich, and F. Zare, "Power system stability and load sharing in distributed generation," in Power System Technology and IEEE Power India Conference, New Delhi, India, Oct. 2008, pp. 1-6.

[8] Y. U. Li and C.-N. Kao, "An accurate power control strategy for powerelectronics-interfaced distributed generation units operating in a lowvoltage multibus microgrid," IEEE Transactions on Power Electronics, vol. 24, no. 12, pp. 2977-2988, 2009.

[9] J. M. Guerrero, J. C. Vasquez, J. Matas, M. Castilla, and L. G. de Vicuna, "Control strategy for flexible microgrid based on parallel line-interactive UPS systems," IEEE Transactions on Industrial Electronics, vol. 56, no. 3, pp. 726-736, 2009.

[10] J. W. Simpson-Porco, F. Dörfler, and F. Bullo, "Synchronization and power sharing for droop-controlled inverters in islanded microgrids," Automatica, Nov. 2012, provisionally accepted.

[11] Z. Wang, M. Xia, and M. D. Lemmon, "Voltage stability of weak power distribution networks with inverter connected sources," in American Control Conference, Washington DC, USA, 2013, submitted.

[12] J. Schiffer, A. Anta, T. D. Trung, J. Raisch, and T. Sezi, "On power sharing and stability in autonomous inverter-based microgrids," in IEEE Conf. on Decision and Control, Maui, HI, USA, Dec. 2012, pp. $1105-1110$

[13] M. Andreasson, H. Sandberg, D. V. Dimarogonas, and K. H. Johansson, "Distributed integral action: Stability analysis and frequency control of power systems," in IEEE Conf. on Decision and Control, Maui, HI, USA, Dec. 2012, pp. 2077-2083.

[14] Q. Shafiee, J. C. Vasquez, and J. M. Guerrero, "Distributed secondary control for islanded microgrids - A networked control systems approach," in Annual Conference on IEEE Industrial Electronics Society, Montréal, Canada, Oct. 2012, pp. 5637-5642.

[15] N. Biggs, "Algebraic potential theory on graphs," Bulletin of the London Mathematical Society, vol. 29, no. 6, pp. 641-683, 1997.

[16] L. O. Chua, C. A. Desoer, and E. S. Kuh, Linear and Nonlinear Circuits. McGraw-Hill, 1987.

[17] P. Kundur, Power System Stability and Control. McGraw-Hill, 1994

[18] F. Dörfler, M. Chertkov, and F. Bullo, "Synchronization in complex oscillator networks and smart grids," Proceedings of the National Academy of Sciences, vol. 110, no. 6, pp. 2005-2010, 2013.

[19] F. Dörfler and F. Bullo, "Kron reduction of graphs with applications to electrical networks," IEEE Transactions on Circuits and Systems I: Regular Papers, vol. 60, no. 1, pp. 150-163, 2013.

[20] J. W. Simpson-Porco, F. Dörfler, and F. Bullo, "Voltage stabilization in microgrids using quadratic droop control," in IEEE Conf. on Decision and Control, Florence, Italy, Dec. 2013, submitted. 